\title{
Evaluation of the Cytotoxicity and Immune and Subacute Toxicity of Camptothecin-loaded Nanoparticles
}

\author{
Hong $\mathrm{T}^{1,2 \#}$, Li SM ${ }^{3 \#}$, Huang $\mathrm{Y}^{1,2}$, Shu $\mathrm{K}^{1}$, Li LX ${ }^{1,2}$, Liu L ${ }^{4}$, Liu $\mathrm{ZY}^{1,2^{*}}$ \\ ${ }^{1}$ Experimental Animal Center, Jiangxi University of Traditional Chinese Medicine, Nanchang, China; ${ }^{2}$ Key Laboratory of Pharmacology \\ of Traditional Chinese Medicine in Jiangxi, Nanchang, China; ${ }^{3}$ Jiangxi Children Hospital, Nanchang, China; ${ }^{4}$ Jiangxi Province Center of \\ Health Guidance, Nanchang, China \\ \#Contributed equally to this paper.
}

\begin{abstract}
CPT-loaded NPs with polyethylene glycol shells can increase its solubility in water and remain in circulation for a long time. This experiment has evaluated cytotoxicity and immune and subacute toxicity of CPT NPs. Camptothecin (CPT) was incorporated into biotin-F127-PLA or F127-PLA polymeric nanoparticles; a dialysis method was used for non-targeted CPT NPs and anti-CA125 antibodies for targeted CPT NPs. A cytotoxicity test was also conducted based on the growth inhibition of the mouse fibroblast-like L-929 cell line. The effects of the two CPT-loaded NPs on immunity were also determined through a carbon particle clearance rate assay. Each mouse was intraperitoneally injected with $0.4 \mathrm{ml} / 20 \mathrm{~g}$.bw nanoparticles for 28 consecutive days in the subacute toxicity test. Results have showed that CPT NPs have reduced the toxicity of free CPT on the L-919 cells. The CPT NPs did not induce phagocytosis in the normal mice. At the end of subacute toxicity study no difference was found between the CPT NP and control groups in blood parameter analysis and main organ weight visceral coefficients. These results suggested that the new CPT NPs might elicit low toxic effects at cellular and organism levels.
\end{abstract}

Keywords: Camptothecin; Targeted nanoparticles; Non-targeted nanoparticles; Immune effect; Subacute toxicity

\section{INTRODUCTION}

Camptothecin (CPT) is a natural product derived from the oriental tree Camptotheca acuminata, and this product has been reported to have an antitumor activity from a number of experiments [1]. CPT or its derivatives are effective chemotherapeutic agents for the treatment of patients with cancers, such as cancers in liver [2] breast [3,4] lung [5,6] pancreas [7] ovaries [8] and stomach [9] as well as leukemia and lymphoma [10]. CPT interferes with tumor growth by inhibiting DNA topoisomerase I [11]. CPT represents a new class of chemotherapeutic agents with broad antitumor activity since it is a human topoisomerase I inhibitor, which blocks DNA replication in human cancer cells. However, CPT has limited clinical application against cancer because of its water insolubility and toxic side effects $[12,13]$.

The fact that CPT is difficult to apply is mainly due to its insolubility in water as well as severe side effects. To increase the water solubility of CPT and reduce toxicity, researchers developed various CPT delivery systems, including organic non-CPT compounds. Chemicals such as Indolocarbazoles, indenoisoquinolines, and dibenzonaphthyridines have been used in clinical settings [14]. Other delivery systems including pulmonary targeting microparticulate CPT delivery system [15] and nanoparticles (NPs). Among drug delivery systems, polymeric NPs have been extensively investigated because of their unique characteristics. However, the majority of polymer drug carriers have passive targeting characteristics. As such, their clinical application in cancer treatment is limited.

CPT-loaded NPs with polyethylene glycol shells can remain in circulation for a long duration [16,17], thereby prolonging the exposure of tumor cells to antitumor drugs. These drugs subsequently reach their target site during passive targeting through enhanced permeability and retention. However, CPT in passive targeting delivery still has numerous challenges associated with low target-tissue concentrations and unpredictable toxicity. For example, topotecan and irinotecan CPT analogues have been used clinically, but the hematological toxicity of these drugs is still common, including leukopenia, thrombocytopenia and haemoglobin reduction.

In our preliminary study, CPT-loaded biotinylated Pluronic F127/

Correspondence to: ZhiYong Liu, Experimental Animal Center, Jiangxi University of Traditional Chinese Medicine, Nanchang 330004, China, Tel: +86 13307091857; E-mail: liuzhiyong0791@163.com

Received: December 24, 2018; Accepted: January 11, 2019; Published: January 18, 2019

Citation: Hong T, Li SM, Huang Y, Shu K, Li LX, et al. (2019) Evaluation of the Cytotoxicity and Immune and Subacute Toxicity of Camptothecin-loaded Nanoparticles. Pharm Anal Acta 10:604. doi: 10.35248/2153-2435.19.10.604

Copyright: @ 2019 Hong T, et al. This is an open-access article distributed under the terms of the Creative Commons Attribution License, which permits unrestricted use, distribution, and reproduction in any medium, provided the original author and source are credited. 
polylactic acid (biotin-F127-PLA) NPs were prepared via dialysis for non-targeted CPT NPs. The targeted CPT NPs were prepared by conjugating the biotin-F127-PLA NPs with anti-CA125 antibodies. In vitro release and cytotoxicity tests were also conducted. The tissue distribution, antitumor effect in vivo, carbon particle clearance rate, and expression of survivin gene were investigated after the CPT-loaded NPs were intravenously (i.v.) administered. The in vitro release of the targeted CPT NPs exhibited a $40 \%$ initial burst within $12 \mathrm{~h}$, followed by a slow release. The cytotoxicity test performed on $\mathrm{H} 22$ cells showed that the in vitro antitumor effects of the CPTloaded targeted NPs were stronger than those of the CPT-loaded non-targeted NPs and the free CPT. The targeted NPs could reduce the side effects of CPT during immunosuppression. The highest CPT concentration was found in the liver of H22 cell-bearing mice that received i.v. injection of the CPT-loaded NPs. The in vivo antitumor effects of the targeted CPT NPs were superior to those of the free CPT and the non-targeted CPT NPs, which might be associated with the reduced mRNA expression of survivin during the treatment period.

Antitumor drugs often affect normal cells while eradicating tumor cells. Pluronic is a commercial family of macromolecular surfactants with the chemical name poly(ethylene oxide)-poly(propylene oxide)poly(ethylene oxide) (PEO-PPO-PEO). It is biocompatible to some Pluronic products, such as Pluronic F127, a substance high in PEO content and has been approved by the U.S. Food and Drug Administration for use in biomedical materials and drug carriers. This material shows a reversed thermal behavior: it appears as fluid at refrigerator temperature, but it becomes a soft gel at body temperature. In vitro release experiments indicated that Pluronic gel may serve as a rate-controlling barrier and can be useful as a vehicle for the sustained-release preparations of drugs for intraperitoneal (i.p.) administration. Pluronic acid F-127 blocks the permeabilizing effect of CSA-13 on the plasma membrane of human umbilical vein endothelial cells without modifying its toxic effects on mitochondrial functions [18]. Pluronic F-127 (PLF-127) gels have been evaluated as a sustained-release vehicle for the intraperitoneal administration of mitomycin $\mathrm{C}$ (MMC) to enhance the therapeutic effects of MMC against sarcoma-180 ascite tumor in mice. The lifespan of tumor-bearing mice is prolonged after therapeutic PLF-127 is injected. PLF-127 containing MMC is therapeutically more active than its free counterpart. MMC in PLF-127 has a high chemotherapeutic efficiency at high doses, which causes MMC to become toxic if it is used alone [19]. Moreover, Pluronic acid F-127 decreased the toxicity of CSA-13 against eukaryotic cells [18]. However, the cytotoxicity of CPT-loaded NPs and their effects on the immune function of normal animals is yet to be explored. The goal of this experiment was to investigate the toxicity of CPT-loaded NPs as well as increasing their safety level in human application for the treatment of various ailments.

\section{MATERIALS AND METHODS}

\section{Materials}

CPT was kindly provided by Beijing Hui Tak Technology Ltd. Amphiphilic block copolymers, namely, Pluronic-PLA and biotin-pluronic-PLA, were synthesized through ring-opening polymerization and provided by Dr. Xiangyuan Xiong from the Jiangxi Normal University of Science and Technology [20]. L-929 cell lines were provided by the Shanghai Cell of Chinese Academy of Sciences. For the cell culture medium, 10\% fresh calf serum was from Hangzhou Siji Bioengineering Materials Research Institute,
DMEM was acquired from Gibco, and the biotinylated anti-CA125 antibody MAB X306 was purchased from Hytest (Turku, Finland). Male ICR mice $(20 \pm 2 \mathrm{~g})$ were obtained from Hunan SLAC Experiment Animal Ltd. (Changsha, China). In this experiment, a barrier housing facility was used in accordance with the National Standard Laboratory Animal Requirements of Environment and Housing Facilities (GB 14925-2010). The care of laboratory animal and the animal experimental operation were performed in accordance with the committee of the Jiangxi University of Traditional Chinese Medicine (2016JZ029). No contaminants, which would have interfered with or affected the results of the study, were present in the feed or water. All other chemicals were of reagent grade.

\section{Preparation and characterization of CPT-loaded NPs}

Aqueous CPT-loaded F127-PLA NPs were prepared using the same method as the F127-PLA block copolymer [20]. The aggregates of a typical Biotin-F127-PLA-61 in aqueous solutions were prepared as follows: Biotin-F127-PLA-61 block copolymer (12 mg) and CPT (1 mg) were dissolved in THF and then the mixture solution was added drop wise to ultra-pure water $(15 \mathrm{~g})$ under gentle stirring. The polymer aggregated solution was dialyzed against ultra-pure water for $5 \mathrm{~h}$ using a cellulose membrane bag (molecular weight cut-off 12,000-14,000 Da) to remove THF outside biotin-F127PLA-87 aggregate. The water was exchanged at intervals of 1 h. The final concentration of Biotin-F127-PLA-87 aggregates is about 0.08 wt $\%$. The other Biotin-Pluronic-PLA nanoparticles in aqueous were prepared in the same way. Targeted CPT NPs were made by conjugating biotinylated drug-loaded NPs with anti-CA125 antibodies. The preparation of targeted drug-loaded nanoparticles was done by conjugating biotinylated drug-loaded nanoparticles with anti-CA125 antibody. The biotinylated drugloaded nanoparticles $(0.5 \mathrm{nmoL})$ were diluted with PBS. The nanoparticles were incubated in excessive streptavidin at $37^{\circ} \mathrm{C}$ for $30 \mathrm{~min}$ and washed with PBS for three times to remove unreacted streptavidin. Biotinylated anti-CA125 antibody, MAB X306, were then added to the nanoparticles and incubated at $37^{\circ} \mathrm{C}$ for $30 \mathrm{~min}$, washed with PBS three times (each time $3 \mathrm{~min}$ ), and washed with deionized water for $1 \mathrm{~min}$.

\section{In vitro toxicity of CPT NPs}

The test applied a cytotoxicity test based on the growth inhibition of mouse fibroblast-like L-929 cell line [21-23]. This method was used to culture mouse fibroblast L-929 cells with targeted and nontargeted CPT NPs for 2, 4 and 7 days. Cell growth was observed under a microscope. The relative proliferation rate (RGR) of the cell was calculated and referenced using the following formula: cell $\mathrm{RGR}=\mathrm{OD}$ of the experimental group/negative control group $\times 100 \%$. RGRs were calculated to quantitatively evaluate the materials that might be toxic to the cells. According to the classification standard, RGRs were divided into levels $\mathrm{O}-\mathrm{V}$ to assess material toxicity level: 100\% RGR or higher for level 0, 75\%-99\% RGR for level I, 50\%-74\% RGR for level II, 25\%-49\% RGR for level III, 1\%-24\% RGR for level IV, and 0\% RGR for level V.

Preparation of cell suspension: The L-929 cells cultured for $72 \mathrm{~h}$ and grown vigorously were treated with trypsin after 5-10 min of their digestion. The cells could detach from the bottle wall and mix with cell culture fluid to facilitate counting. Finally, the cell concentration was adjusted to 10,000 cells $/ \mathrm{ml}$ in the cell culture medium. 
2.3.2 Each bottle was added with $3 \mathrm{ml}$ of the cell suspension, inverted, and incubated in a $\mathrm{CO}_{2}$ incubator for $24 \mathrm{~h}$.

Test fluid exchange: After $24 \mathrm{~h}$, the media in all bottles was discarded in a cell culture flask. The negative control group was replaced with a fresh cell culture medium. The test liquid group was also replaced by a fresh culture medium containing $50 \%$ of the test liquid. The culturing was resumed at $37^{\circ} \mathrm{C}$.

Cell morphology observation and counting: The cells were observed and counted under an inverted microscope on the $2^{\text {nd }}$, $4^{\text {th }}$, and $7^{\text {th }}$ days after the medium was changed. To calculate the cell concentration, the cells were digested with trypsin to prepare a cell suspension. The number was then counted under a microscope with a hemocytometer.

\section{In vivo effects of CPT NPs on the immune system of normal mice}

The ICR mice were injected with free CPT, non-targeted CPT NPs, and targeted CPT NPs consecutively for 14 days, weighed, and injected with Indian ink in the tail vein $(0.1 \mathrm{ml} / 10 \mathrm{gbw})$. At $2 \mathrm{~min}\left(\mathrm{t}_{1}\right)$ and $10 \mathrm{~min}\left(\mathrm{t}_{2}\right)$ post-injection, approximately $50 \mu \mathrm{l}$ of blood was collected from the eyes. About $20 \mu \mathrm{l}$ of blood was then immediately added into $2 \mathrm{ml}$ of $0.1 \% \mathrm{Na}_{2} \mathrm{CO}_{3}$ solution. The optical densities (OD) of the dilutions at $2 \mathrm{~min}\left(\mathrm{OD}_{1}\right)$ and $10 \mathrm{~min}$ $\left(\mathrm{OD}_{2}\right)$ were measured at $680 \mathrm{~nm}$ using a spectrophotometer. After the mice were sacrificed through cervical dislocation, the weights of the liver and spleen were measured. The granule removal $(K)$ and the phagocyte index $(\alpha)$ were calculated as follows:

$$
\begin{gathered}
K=\frac{\log O D_{1}-\log O D_{2}}{t_{2}-t_{1}} \\
\alpha=\frac{\text { Body weight }}{\text { Liver weight }+ \text { Spleen weight }} \times \sqrt[3]{K}
\end{gathered}
$$

\section{Subacute toxicity of CPT NPs in normal mice}

Twenty-four male ICR mice were divided into four groups ( $n=6$ each group): 1 control group, 1 free CPT group, and 2 CPT NP groups. Each mouse was administered with $0.4 \mathrm{ml} / 20 \mathrm{~g}$.bw nanoparticles via i.p. injection for 28 consecutive days.

Clinical observation and mortality during the study period: All of the mice were observed daily for clinical signs of toxicity, morbidity, and mortality. Detailed clinical observations were recorded. The mice from each group were weighed once every 4 days.

Clinical pathology: At the end of the study, mice were anesthetized with carbon dioxide, followed by an overnight fast. The mice's blood samples were obtained through retro-orbital bleeding. Hematology measurements included white blood cell (WBC), red blood cell (RBC), hemoglobin (HGB), hematocrit (HCT), lymphocyte (LYM), granulocyte RDW, and platelet (PLT). Clinical chemistry measurements covered total bilirubin (BIL-T), alanine aminotransferase (ALT), aspartate aminotransferase (ALB), globulin (GLB), alkaline phosphatase (ALP), urea nitrogen (BUN), creatinine (CREA), potassium $(\mathrm{K})$, sodium $(\mathrm{Na})$, and chlorine $(\mathrm{Cl})$.

Urinalysis: At the end of the study, each mouse was subjected to urinalysis, and results were read using a Uritest-180 urine analyzer. The measured parameters were appearance (color and clarity), glucose (uGLU), bilirubin (uBIL), ketone (KET), occult blood $(\mathrm{RBC})$, acidity $(\mathrm{pH})$, protein $(\mathrm{PRO})$, urobilinogen ( $\mathrm{uBG})$, nitrite (NIT), and leukocytes (LEU).

Necropsy: All animals in the study were subjected to a detailed gross necropsy that included careful examination of the external surface of the body, all the orifices, cranial, thoracic, and abdominal cavities, as well as their contents. The control and high-dose groups were subjected to gross necropsy after an observation period of 14 days. The liver, kidneys, adrenals, testes, epididymis, uterus, ovaries, thymus, spleen, brain, and heart of all the mice were trimmed of any adherent tissue as appropriate, and their wet weight was taken as soon as possible. Organ coefficients were calculated according to organ weights and body weights. The following tissues were preserved in the most appropriate fixation medium for histopathologic examination: all gross lesions, brain (representative regions, including the cerebrum, cerebellum, and medulla/pons), spinal cord, stomach, thyroid, thymus, small and large intestines, liver, kidneys, adrenals, pancreas, spleen, heart, trachea, lungs (preserved by inflating with a fixative and then immersed), gonads, uterus, accessory sex organs, prostate, urinary bladder, lymph nodes, peripheral nerve (sciatic or tibial) preferably in proximity to the muscle, and a section of the bone marrow. Clinical and other findings might suggest the need to examine additional tissues. The relative organ weights were calculated according to the following formula: relative organ weight $(\%)=$ weight of organ $(\mathrm{g}) /$ body weight $(\mathrm{g}) \times 100$.

Histopathologic examination: Tissue and organ (all gross lesions, brain, spinal cord, stomach, thyroid, thymus, small and large intestines, liver, kidneys, adrenals, spleen, heart, trachea and lungs, gonads, uterus, accessory sex organs, prostate, urinary bladder, lymph nodes, peripheral nerve, and bone marrow) samples from the control and treated mice were cut into thin slices and then fixed with $4 \%$ formaldehyde for more than 1 week. The tissues and organs were then processed through a graded series of ethanol and xylene and embedded in paraffin. Organ and tissue sections were stained with hematoxylin and eosin (H\&E) for histopathological examination under a microscope, and abnormal conditions were recorded.

A complete histopathological examination was carried out on the preserved organs and tissues of all mice in the control and highdose groups. This examination was extended to the mice in other dosage groups if treatment-related changes were observed in the high-dose group.

\section{Statistical analysis}

Data were presented as means \pm SD and examined for normal distribution by performing homogeneity of variance. One-way ANOVA was conducted to analyze the data with homogeneity of variance. Each group was compared with the control group via Dunnett's $\mathrm{t}$ test. If the data were abnormally distributed or had heterogeneity of variance, Wilcoxon-Wilcox rank sum test was used instead. Fisher's exact probability test was used to enumerate data. The results of urinalysis from the reagent strips were analyzed using Kruskal-Wallis test, followed by multiple comparisons with Dunnett's t test. Clinical signs necropsy findings, and histopathological findings were represented as frequencies and subjected to Fisher's exact probability test as appropriate. The significant probability values $p<0.05^{(*)}$ or $p<0.01^{(*)}$ were represented as asterisks. 


\section{RESULTS AND DISCUSSION Cytotoxicity of CPT NPs}

On the $2^{\text {nd }}$ and $4^{\text {th }}$ days, the L-929 cells of the targeted and nontargeted CPT NP groups were adherent and had triangular or irregular shapes. In spite of this, the growth experienced no suppression or interruption. On the $7^{\text {th }}$ day, the cells grew well and showed normal morphology. They were adherent and had a columnar or irregular triangular shape. The suspension cells of these two groups in the test solution were slightly higher than those of the negative control group. The CPT group exhibited significantly lower growth than the targeted and non-targeted CPT NPs groups did on the $2^{\text {nd }}, 4^{\text {th }}$, and $7^{\text {th }}$ days. The CPT group had significantly higher cell death than the two CPT NP groups. CPT NPs had grade 1 cytotoxicity, whereas free CPT had grade 2 (2 and 4 days). After 7 days, the cytotoxicity of CPT NPs remained at grade 1 , whereas the cytotoxicity of the free CPT reached grade 3 (Table 1).

\section{Effect of NPs on the carbon particle clearance of mice}

Immunoregulation is a complex balance in the body and any imbalance in the immunological mechanism would lead to pathogenesis. Immunity is suppressed in cancer. Many free anticancer drugs used in chemotherapy have strong side effects that deteriorate the immune system. The phagocytic capacity of peritoneal macrophages is one of the parameters that measure the nonspecific immune function of the body, and this capacity is assessed by a carbon clearance test. Table 2 shows that the clearance rate of charcoal particles increased in the free CPT group. However, the carbon clearance rate of the CPT NP group did not significantly differ from that of the control mice. The CPT NPs did not enhance phagocytosis in the normal mice, and the difference comparing to free CPT was significant (p). No significant difference was observed when these findings were compared with those of the control group. This result might be attributed to the high concentration of CPT NPs in the target tissue and their low side effects (Table 2).

\section{Subacute toxicity of CPT NPs}

Clinical signs and mortality: There was no treatment-related mortality was observed in the mice treated with CPT NPs for 4 weeks at any of the dosages tested. Also no treatment-related clinical findings were recorded in each dose group.

Changes in body weight: There was no difference was observed in the body weight gain between the CPT NP group and the nontarget CPT NP group compared with that of the control group. However, the body weight of the free CPT group was significantly lower than data from the two other CPT groups and the control group $(\mathrm{p}<0.05)$ (Figure 1).

Hematologic examination: Blood parameter analysis is essential for the evaluation of hematological lesions. The subacute toxicity of CPT NPs was examined 28 days after the drug was administered. The total number of WBC was significantly different between the free CPT and those CPT NP groups $(p<0.01)$. There was no

Table 1: Cytotoxicity of CPT NPs of the mice fibroblast-like L-929 cell line.

\begin{tabular}{|c|c|c|c|c|c|c|}
\hline \multirow{2}{*}{ Groups } & \multicolumn{2}{|c|}{$2 \mathrm{~h}$} & \multicolumn{2}{|c|}{$48 \mathrm{~h}$} & \multicolumn{2}{|c|}{$72 \mathrm{~h}$} \\
\hline & RGR (\%) & Toxicity level & RGR (\%) & Toxicity level & RGR (\%) & Toxicity level \\
\hline Control & 100 & 0 & 100 & 0 & 100 & 0 \\
\hline $\mathrm{CPT}$ & 60.13 & 2 & 54.26 & 2 & 45.33 & 3 \\
\hline Non-targeted CPT NPs & 81.53 & 1 & 89.21 & 1 & 91.62 & 1 \\
\hline Targeted CPT NPs & 83.27 & 1 & 90.69 & 1 & 94.15 & 1 \\
\hline
\end{tabular}

Table 2: Effects of CPT NPs on the carbon particle clearance in mice.

\begin{tabular}{ccc}
\hline Group & Carbonaceous granule removal (K) & Phagocytic index (a) \\
\hline Control & $0.028 \pm 0.011$ & $3.49 \pm 0.72$ \\
\hline Free CPT & $0.042 \pm 0.016^{*}$ & $5.17 \pm 0.63^{*}$ \\
\hline Non-targeted CPT NPs & $0.030 \pm 0.013^{*}$ & $3.89 \pm 0.51^{*}$ \\
\hline Targeted CPT NPs & $0.026 \pm 0.009^{*}$ & $3.21 \pm 0.32^{*}$ \\
\hline
\end{tabular}

${ }^{*} \mathrm{p}<0.05$ compared with the control group; ${ }^{*}$ with free CPT group, $\mathrm{p}<0.05$

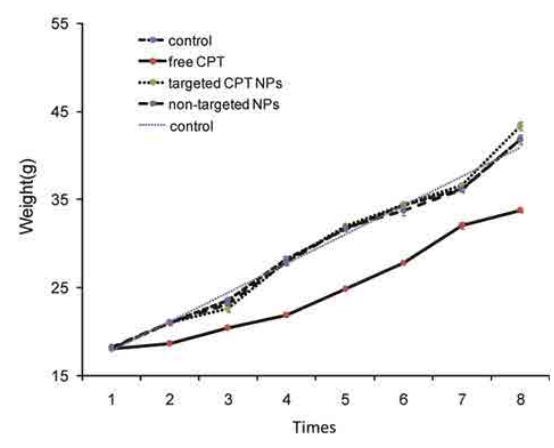

Figure 1: The body weight of subacute toxicity of CPT NPs. No difference was observed in the body weight gain between the CPT NPs group and the non-target CPT NPs group compared with that of the control group. However, the body weight of the free CPT group was significantly lower than that of the two other CPT groups and the control group $(\mathrm{p}<0.05)$. 
Table 3: Results of the hematological examination in male mice during the acute toxicity test with CPT NPs $(\bar{X} \pm S, n=6)$.

\begin{tabular}{|c|c|c|c|c|}
\hline Check project & $\mathrm{CPT}$ & Targeted CPT NPs & Non-targeted CPT NPs & Control group \\
\hline Total white blood Cell count $\left(\times 10^{9} / \mathrm{L}\right)$ & $2.95 \pm 1.25^{\# \#}$ & $7.42 \pm 2.20^{* *}$ & $7.24 \pm 1.59^{* *}$ & $7.36 \pm 0.84^{* *}$ \\
\hline Hemoglobin $(\mathrm{g} / \mathrm{L})$ & $131.2 \pm 44.06$ & $141.00 \pm 9.06$ & $135.00 \pm 7.52$ & $158.2 \pm 44.06$ \\
\hline Red blood cells $\left(\times 10^{12} / \mathrm{L}\right)$ & $8.24 \pm 2.42$ & $9.26 \pm 0.48$ & $8.76 \pm 0.38$ & $10.28 \pm 2.49$ \\
\hline Hematocrit & $31.76 \pm 4.24^{\#}$ & $46.92 \pm 3.14^{*}$ & $44.34 \pm 2.88^{*}$ & $53.44 \pm 3.49^{*}$ \\
\hline Mean corpuscular volume & $41.22 \pm 1.26^{\#}$ & $50.74 \pm 2.60^{*}$ & $50.66 \pm 1.74^{*}$ & $51.22 \pm 1.32^{*}$ \\
\hline Mean corpuscular hemoglobin & $16.26 \pm 0.41$ & $15.18 \pm 0.58$ & $15.36 \pm 0.56$ & $15.02 \pm 0.49$ \\
\hline $\begin{array}{l}\text { Mean corpuscular hemoglobin } \\
\text { concentration }\end{array}$ & $301.00 \pm 2.45$ & $300.00 \pm 8.06$ & $304.00 \pm 4.47$ & $303.21 \pm 7.34$ \\
\hline Red cell distribution width & $13.64 \pm 0.98$ & $14.12 \pm 1.30$ & $15.44 \pm 1.52$ & $14.51 \pm 1.41$ \\
\hline Platelet & $835.60 \pm 179.50$ & $717.20 \pm 102.33$ & $752.80 \pm 154.31$ & $821.45 \pm 132.12$ \\
\hline Mean platelet volume & $6.10 \pm 0.27$ & $5.92 \pm 0.28$ & $5.78 \pm 0.19$ & $6.02 \pm 0.23$ \\
\hline Platelet distribution width & $14.72 \pm 0.19$ & $14.68 \pm 0.26$ & $14.60 \pm 0.23$ & $14.27 \pm 0.21$ \\
\hline Thrombocytocrit & $0.37 \pm 0.21$ & $0.43 \pm 0.07$ & $0.44 \pm 0.10$ & $0.41 \pm 0.09$ \\
\hline
\end{tabular}

*with free CPT group, $\mathrm{p}<0.05$; " with free CPT group, $\mathrm{p}<0.01$; " with control group, $\mathrm{p}<0.05$; "\# with control group, $\mathrm{p}<0.01$

Table 4: Organ coefficients of CPT NPs during the subacute toxicity test in mice $(\bar{X} \pm S, n=6)$.

\begin{tabular}{ccccc}
\hline $\begin{array}{c}\text { Organ coefficient } \\
\text { (\% body weight })\end{array}$ & Control group & Targeted CPT NPs & Non-targeted CPT NPs & free CPT \\
\hline Cerebellum & $0.32 \pm 0.06$ & $0.40 \pm 0.06$ & $0.32 \pm 0.07$ & $0.29 \pm 0.04$ \\
\hline Brain & $0.96 \pm 0.06$ & $1.12 \pm 0.14$ & $1.03 \pm 0.11$ & $1.05 \pm 0.10$ \\
\hline Heard & $0.44 \pm 0.26$ & $0.50 \pm 0.08$ & $0.47 \pm 0.02$ & $0.43 \pm 0.09$ \\
\hline Lung & $0.67 \pm 0.05$ & $0.73 \pm 0.14$ & $0.65 \pm 0.10$ & $0.61 \pm 0.08$ \\
\hline Liver & $4.54 \pm 0.24$ & $4.60 \pm 0.85$ & $4.62 \pm 0.70$ & $2.87 \pm 0.86^{*}$ \\
\hline Spleen & $0.29 \pm 0.02^{*}$ & $0.31 \pm 0.14^{*}$ & $0.36 \pm 0.17^{*}$ & $0.17 \pm 0.13^{*}$ \\
\hline Kidney & $1.56 \pm 0.12$ & $1.62 \pm 0.28$ & $1.63 \pm 0.15$ & $1.49 \pm 0.13$ \\
\hline Testis & $0.81 \pm 0.15$ & $0.97 \pm 0.29$ & $0.85 \pm 0.12$ & $0.76 \pm 0.13$ \\
\hline Epididymis & $0.82 \pm 0.28^{* *}$ & $0.87 \pm 0.13^{* *}$ & $0.82 \pm 0.11^{* *}$ & $0.41 \pm 0.14^{\# \#}$ \\
\hline
\end{tabular}

"with free CPT group, $\mathrm{p}<0.05$; " with free CPT group, $\mathrm{p}<0.01$; ${ }^{*}$ with control group, $\mathrm{p}<0.05$; "\# ${ }^{*}$ ith control group, $\mathrm{p}<0.01$

significant difference was observed between the targeted CPT NPs group and the control group. The two CPT NP groups had no effects on the number of RBCs. A difference was observed in the average volume and hemoglobin content of RBCs between the CPT NP and free CPT groups, but no difference was found between the CPT NP and control groups (Table 3).

Serum biochemistry and urinalysis: Serum biochemistry and urinalysis were performed to assess the overall health status of the mice and detect alterations in metabolic processes. The biochemical indices in each group showed no significant changes compared with those of the control group.

Organ weights: Relative organ weight is an important index that determines whether an organ has been exposed to injury. At the end of the experiment, no notable abnormality was detected in the gross anatomy of the organs of each dose group. The visceral coefficients of the liver $(p>0.05)$ and spleen $(p>0.05)$ were no significantly higher in the nanometer vesicles with CPT than in the control group. Additionally, the weight of the two organs significantly decreased in the free CPT group compared with that in the control group. No change was observed in the weight of both testicles; however, the epididymal visceral coefficient of free CPT group $(p<0.05)$ was significantly lower than two CPT NP and control groups. The reduction in the weight of the epididymis has likely affected sperm storage (Table 4), which may be a worthwhile phenomenon to conduct in depth studies on in the future.

\section{DISCUSSION AND CONCLUSIONS}

CPT was incorporated into biotin-F127-PLA or F127-PLA polymeric NPs through a dialysis method. The targeted NPs were prepared by conjugating biotin-F127-PLA NPs with anti-CA125 antibodies. Results of this experiment indicated that the new targeted CPT NPs may reduce the toxicity of cellular and organism level.

Most human nanoparticle preparations are intravenously injected. In this experiment, intraperitoneal injection is used. The drug is administered through portal vein or inferior vena cava into the systemic circulation, and there is a first-pass elimination effect, which may lead to intraperitoneal administration cannot reach the level of intravenous administration. Therefore, the different effects of the two methods need to be further observed in future experiments.

\section{ACKNOWLEDGEMENTS}

This work was supported by the Key Science and Technology of Jiangxi Province Education Department (Project No. GJJ150830), the Science and Technology Supported Program of Jiangxi Province (Project No. 20111BBF60025), and the Supported Program of Jiangxi University of Traditional Medicine. 


\section{REFERENCES}

1. Ardizzoni A. Camptothecin analogues in the treatment of non-small cell lung cancer. Lung Cancer. 1995;12:S177-S185.

2. Yan JB. Analysis of the effect of camptothecin as an adjunct treatment of primary liver cancer (author's transl). Zhonghua Wai Ke Za Zhi. 1980;18:361-362.

3. Clements MK, Wasi S, Daoud SS. Camptothecin exhibits selective cytotoxicity towards human breast carcinoma as compared to normal bovine endothelial cells in vitro. Anticancer Drugs. 1996;7(8):851-857.

4. Miller KD, Soule SE, Haney LG, Guiney P, Murry DJ. A phase II study of 9-nitro-camptothecin in patients with previously treated metastatic breast cancer. Invest New Drugs. 2004;22:69-73.

5. Fukuoka M, Niitani H, Suzuki A, Motomiya M, Hasegawa K. A phase II study of CPT-11, a new derivative of camptothecin, for previously untreated non-small-cell lung cancer. J Clin Oncol. 1992;10(1):16-20.

6. Ardizzoni A, Oncologvl I, Ricema N. Camptothecin analogues in the treatment of non-small cell lung cancer. Cancer. 1995;13:185-232.

7. Tedesco KL, Berlin J, Rothenberg M, Choy H, Wyman K. A phase I study of concurrent 9-nitro-20(s)-camptothecin (9NC/Orathecin) and radiation therapy in the treatment of locally advanced adenocarcinoma of the pancreas. Radiother Oncol. 2005;76(1):54-58.

8. Tanaka T, Umesaki N, Ogita S. Camptothecin and mitomycin combination chemotherapy on ovarian clear cell carcinoma with multiple systemic metastases. Eur J Gynaecol Oncol. 2000;21:377-379.

9. Litvak DA, Papaconstantinou HT, Hwang KO, Kim M, Evers BM. Inhibition of gastric cancer by camptothecin involves apoptosis and multiple cellular pathways. Surgery. 1999;126(2):223-230.

10. Ohno R, Okada K, Masaoka T, Kuramoto A, Arima T. An early phase II study of CPT-11: a new derivative of camptothecin, for the treatment of leukemia and lymphoma. J Clin Oncol. 1990;8:1907-1912.

11. Beretta GL, Gatti L, Perego P, Zaffaroni N. Camptothecin resistance in cancer: insights into the molecular mechanisms of a DNA-damaging drug. Curr Med Chem. 2013;20:1541-1565.

12. Hartmann JT, Lipp HP. Camptothecin and podophyllotoxin derivatives: inhibitors of topoisomerase I and II-mechanisms of action, pharmacokinetics and toxicity profile. Drug Saf. 2006;29:209-230.
13. Zhou L, Du L, Chen X, Li X, Li Z. The antitumor and antimetastatic effects of $\mathrm{N}$-trimethyl chitosan-encapsulated camptothecin on ovarian cancer with minimal side effects. Oncol Rep. 2010;24:941-948.

14. Gokduman k. Strategies targeting dna topoisomerase I in Cancer Chemotherapy: Camptothecins, Nanocarriers for Camptothecins, Organic Non-Camptothecin Compounds and Metal Complexes. Curr Drug Targets. 2016;17:1928-1939.

15. Chao P, Deshmukh M, Kutscher HL, Gao D, Rajan SS. Pulmonary targeting microparticulate camptothecin delivery system: anticancer evaluation in a rat orthotopic lung cancer model. Anticancer Drugs. 2010;21:65-76.

16. Bildstein L, Dubernet C, Couvreur P. Prodrug-based intracellular delivery of anticancer agents. Adv Drug Deliv Rev. 2011;63:3-23.

17. Luo Z, Jiang J. pH-sensitive drug loading/releasing in amphiphilic copolymer PAE-PEG: Integrating molecular dynamics and dissipative particle dynamics simulations. J Control Release. 2012;162:185-193.

18. Nagant C, Savage PB, Dehaye JP. Effect of pluronic acid F-127 on the toxicity towards eukaryotic cells of CSA-13, a cationic steroid analogue of antimicrobial peptides. J Appl Microbiol. 2012;112:1173-1183.

19. Miyazaki S, Ohkawa Y, Takada M, Attwood D. Antitumor effect of pluronic F-127 gel containing mitomycin C on sarcoma-180 ascites tumor in mice. Chem Pharm Bull. 1992;40:2224-2226.

20. Yang A, Liu Z, Yan B, Zhou M, Xiong X. Preparation of camptothecinloaded targeting nanoparticles and their antitumor effects on hepatocellular carcinoma cell line H22. Drug Delivery. 2016;23:1699. 1706.

21. Jarkelid L, Kjellstrand P, Martinson E, Wieslander A. Toxicity of 20 chemicals from the MEIC programme determined by growth inhibition of L-929 fibroblast-like cells. Altern Lab Anim. 1997;25:55 59.

22. Ray RS, Agrawal N, Sharma A, Hans RK. Use of L-929 cell line for phototoxicity assessment. Toxicol In Vitro. 2008;22:1775-1781.

23. Li J, Chen S, Li W, Yang G, Zhu W. Toxicity of extracts from disposable chopsticks, toothpicks, and paper cups on L-929 cells. Can J Physiol Pharmacol. 2015;93:223-226. 